This information is current as of April 26, 2023.

Geometric and Compositional Appearance of Atheroma in an Angiographically Normal Carotid Artery in Patients with Atherosclerosis

L. Dong, H.R. Underhill, W. Yu, H. Ota, T.S. Hatsukami, T.L. Gao, Z. Zhang, M. Oikawa, X. Zhao and C. Yuan

AJNR Am J Neuroradiol 2010, 31 (2) 311-316 doi: https://doi.org/10.3174/ajnr.A1793

http://www.ajnr.org/content/31/2/311 


\section{ORIGINAL RESEARCH}

L. Dong

H.R. Underhill

W. Yu

H. Ota

T.S. Hatsukami

T.L. Gao

Z. Zhang

M. Oikawa

X. Zhao

C. Yuan

\title{
Geometric and Compositional Appearance of Atheroma in an Angiographically Normal Carotid Artery in Patients with Atherosclerosis
}

BACKGROUND AND PURPOSE: Arterial remodeling may enable atherosclerotic disease without luminal stenosis. We sought to assess the prevalence and characteristics of atherosclerosis in angiographically normal carotid arteries.

MATERIALS AND METHODS: Forty-six arteries with 0\% stenosis by MRA were evaluated with multicontrast carotid MR imaging at 3T. For each artery, the percentage wall volume (wall volume/[lumen volume + wall volume] $\times 100 \%$ ) and the presence versus absence of an LRNC, calcification, IPH, and fibrous cap rupture were recorded. In addition, the relative size of each plaque component (eg, percentage LRNC $=$ LRNC volume/wall volume $\times 100 \%$ ), when present, was calculated.

RESULTS: The mean of percentage wall volume in arteries with $0 \%$ stenosis was $43.0 \pm 6.9 \%$ with a range from $31.6 \%$ to $60.1 \%$. An LRNC was present in $67.4 \%(31 / 46)$ of arteries, calcification was present in $65.2 \%(30 / 46)$, IPH was present in $8.7 \%(4 / 46)$, and fibrous cap rupture was present in $4.3 \%$ (2/46). In arteries with an LRNC $(n=31)$, the average percentage LRNC volume was $8.8 \pm 7.3 \%$ with a range from $1.0 \%$ to $31.5 \%$. For calcification $(n=30)$, the mean percentage calcification volume was $3.8 \pm 4.2 \%$ with a range of $0.1 \%-17.4 \%$. The mean percentage IPH volume $(n=4)$ was $2.7 \pm 1.7 \%$ with a range of $0.5 \%-4.1 \%$.

CoNCLUSIONS: These findings indicate that stenosis by MRA may underestimate the presence of carotid atherosclerosis, and they demonstrate the need for improved methods for accurately identifying carotid atherosclerotic plaque severity.

\begin{abstract}
ABBREVIATIONS: $\mathrm{CB}=$ carotid bulb; $\mathrm{CCA}=$ common carotid artery; $\mathrm{CE}=$ contrast-enhanced; ECST = European Carotid Surgery Trial; ETL = echo-train length; HDL = high-density lipoprotein; $I C A=$ internal carotid artery; ImQ = image quality; $I P H=$ intraplaque hemorrhage; $L D L=$ low-density lipoprotein; LRNC = lipid-rich necrotic core; MIP = maximum intensity projection; MRA = MR angiography; NASCET = North American Symptomatic Carotid Endarterectomy Trial; PDW = proton-attenuation weighted; $\mathrm{SI}=$ signal intensity; $\mathrm{T} 2 \mathrm{~W}=\mathrm{T}$-weighted; $\mathrm{TOF}=$ time-of-flight.
\end{abstract}

$\mathbf{L}$

uminal stenosis is the current standard for assessing the risk of stroke from carotid atherosclerotic disease. ${ }^{1}$ Several large prospective studies have established the value of stenosis measurement for disease severity and the risk of stroke in patients with moderate- and severe-grade symptomatic carotid artery stenosis. ${ }^{2-5}$ However, luminal stenosis may be unreliable in asymptomatic patients and in symptomatic patients with low-grade stenosis. The ECST reported that $43.8 \%$ of the 3018 individuals with symptomatic carotid disease had $<30 \%$ stenosis. $^{6}$ The NASCET found that among patients with $<50 \%$ stenosis, the 5-year rate of any ipsilateral stroke was $22.2 \% .^{3}$ Although each trial used a different reference standard during the stenosis calculation (approximation of the carotid bulb for ECST, distal normal internal carotid artery for NASCET), these findings indicate that regardless of the denominator used, stenosis may be an incomplete measure of atherosclerotic disease severity.

Accordingly, Babiarz et $\mathrm{al}^{7}$ have demonstrated that carotid

Received April 1, 2009; accepted after revision June 15.

From the Departments of Radiology (L.D., H.R.U., H.O., M.O., X.Z., C.Y.) and Surgery (T.S.H.), University of Washington, Seattle, Washington; and Department of Radiology (L.D., W.Y., T.L.G., Z.Z.), Beijing Anzhen Hospital, Capital Medical University, Beijing, China.

Please address correspondence to Chun Yuan, PhD, Vascular Imaging Lab, Department of Radiology, University of Washington, 815 Mercer St, Box 358050, Seattle, WA 98109; e-mail: cyuan@u.washington.edu

DOI 10.3174/ajnr.A1793 atheroma was frequently identified by MR imaging in arteries with minimal angiographic stenosis. In addition, Saam et $\mathrm{al}^{8}$ reported a notably high occurrence (approximately 30\%) of carotid plaques with IPH and fibrous cap rupture in arteries with $\leq 50 \%$ stenosis. In arteries with only $1 \%-15 \%$ stenosis, these features were present in $8.1 \%$ of lesions. ${ }^{8}$ In this study, we sought to extend these previous investigations ${ }^{7,8}$ and determine the prevalence of atherosclerotic disease and plaque compositional features in angiographically normal arteries (ie, $0 \%$ stenosis) identified in subjects with known carotid atherosclerotic disease in the contralateral artery.

\section{Materials and Methods}

\section{Study Population}

Individuals with at least 1 carotid artery with $>50 \%$ stenosis by duplex sonography were serially recruited for MR imaging of their bilateral carotid arteries. At the time of MR imaging, all participants provided answers to a standardized health questionnaire, had their height and weight measured, and underwent phlebotomy. The study procedures and consent forms were reviewed and approved by the institutional review board before study initiation.

\section{MR Imaging Protocol}

Between February 2007 and September 2007, 72 participants were enrolled and underwent imaging on a $3 \mathrm{~T}$ scanner (Signa Excite; 


\begin{tabular}{lc}
\hline Demographics of the sample population & \\
\hline Characteristics & Data \\
\hline Age (yr) & $67 \pm 9.9$ \\
Male sex (\%) & 72.8 \\
Body mass index $\left(\mathrm{Kg} / \mathrm{m}^{2}\right)$ & $25.06 \pm 3.51$ \\
LDL cholesterol $(\mathrm{mmol} / \mathrm{L})$ & $3.31 \pm 1.13$ \\
HDL cholesterol (mmol/L) & $1.04 \pm 0.43$ \\
Active smoker (\%) & 17.5 \\
Hypertension (\%) & 67.6 \\
Diabetes mellitus (\%) & 31.8 \\
History of coronary artery disease (\%) & 17.5 \\
\hline
\end{tabular}

GE Healthcare, Milwaukee, Wisconsin) with bilateral phased array 4-element surface coils. A standardized MR imaging protocol ${ }^{9}$ adapted for imaging at $3 \mathrm{~T}^{10}$ was used to acquire transverse images of the carotid artery centered at the bifurcation of the artery with greater duplex stenosis. 2D T1-weighted images, ${ }^{11}$ 2D multisection double inversion recovery PDW and T2-weighted black-blood images, ${ }^{12}$ and 3D TOF bright-blood angiograms were acquired. Imaging parameters for each weighting were as follows: T1-weighted (TR/TE, 800/11 ms; ETL, 10; 16 sections; scanning time, 5 minutes 58 seconds), T2weighted and PDW (TR/TE, 3500/70 and 12 ms; ETL, 12; 16 sections; scanning time, 3 minutes 40 seconds), and TOF (TR/TE, 21/2.9 ms; flip angle, $15^{\circ}$; 48 sections; scanning time, 2 minutes 4 seconds). All images were obtained with a section thickness of $2 \mathrm{~mm}$, FOV of 140 $\mathrm{mm}$, and matrix size of $512 \times 512$. In addition, a CE T1-weighted sequence $^{11}$ was acquired 5 minutes after administration of 0.2$\mathrm{mmoL} / \mathrm{kg}$ gadopentetate dimeglumine (Magnevist; Bayer Schering Pharma, Berlin, Germany) was injected intravenously by using a power injector at $3 \mathrm{~mL} / \mathrm{s}$ followed by a $20-\mathrm{mL}$ saline bolus at the same speed.
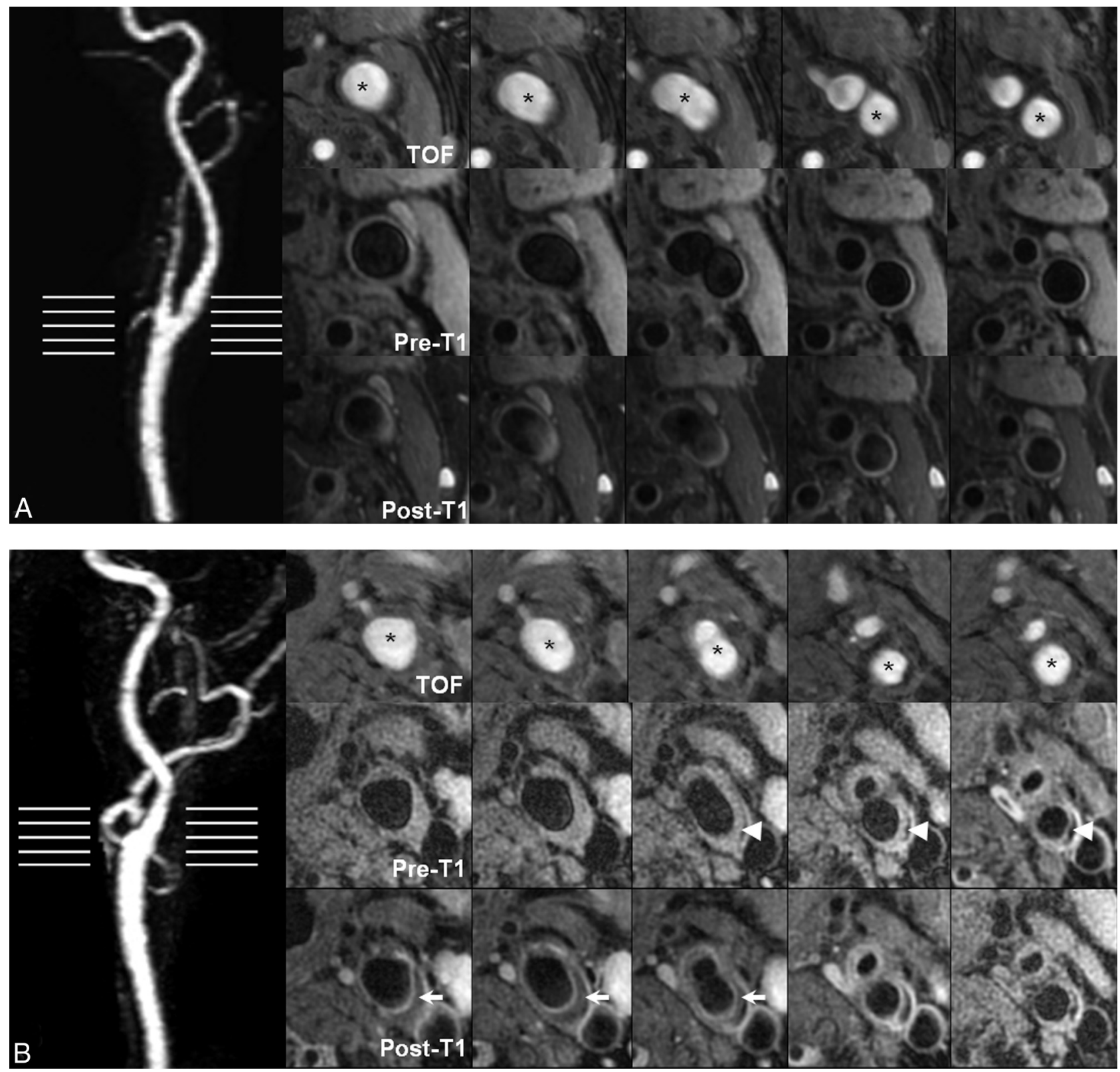

Fig 1. MIP and multicontrast carotid MR images of the left carotid artery from a 67-year-old woman $(A)$ and a 62-year-old man $(B)$. Transverse images are consecutive sections that correspond to horizontal lines on the MRA. $A$, There is $0 \%$ stenosis on MRA and no evidence of plaque formation at any axial location. $B$, There is substantial plaque with evidence of a LRNC (arrows) and calcification (arrowheads), though there is no evidence of stenosis on MRA. The asterisk indicates the lumen of either the CCA or ICA. 
At the time of contrast injection and before the CE T1-weighted acquisition, a 3D CE-MRA was acquired in the coronal plane with a 3D fast-spoiled gradient-recalled echo sequence (TR/TE, 2.5/1.2 ms; flip angle, $40^{\circ}$; section thickness, $2 \mathrm{~mm}$; partitions per 3D slab, 36; matrix, $192 \times 320$; FOV, $320 \mathrm{~mm}$; NEX, 1). $\mathrm{K}$-space was ordered by using a $3 \mathrm{D}$ elliptic centric view order. A fluoroscopic triggering technique was used for detecting the contrast agent bolus. All sequences used the excitation pulses of the manufacturer and were not modified to reduce the specific absorption rate to shorten scanning time.

\section{Image Analysis}

All MRA images were interpreted bilaterally by consensus opinion of 2 trained reviewers blinded to clinical information and transverse carotid MR images. Luminal stenosis was quantified in both carotid arteries by using the NASCET criterion: (1-luminal diameter at the point of maximal narrowing/the diameter of the normal distal internal carotid artery) $\times 100 \% .^{3}$ All diameter measurements were acquired by using GE software (GE Healthcare Advantage workstation, Version 4.2.) and were oriented on MIP images.

For arteries with $0 \%$ stenosis by MRA, multicontrast transverse carotid MR images were interpreted by 2 trained reviewers blinded to clinical information. Each axial section was evaluated for ImQ (4point scale: 1 = poor, $4=$ excellent). Individual sections with ImQ $<2$ were excluded if they were at the proximal or distal coverage of the artery. Sections with ImQ $<2$ in the center of coverage resulted in exclusion of the entire artery. Only consecutive images of an artery with ImQ $\geq 2$ were interpreted. Image-analysis software (CASCADE, ${ }^{13}$ Seattle, Washington) was used to draw the lumen and outer wall boundaries. Lumen volume, wall volume, and total vessel volume (lumen volume + wall volume) were used to calculate percentage wall volume (wall volume/total vessel volume $\times 100 \%$ ), a measure of plaque burden similar to that proposed by Nissen et al ${ }^{14}$ during imaging investigations of the coronary arteries. The presence or absence of calcification, LRNC, IPH, and fibrous cap rupture was determined by using multicontrast imaging criteria that have been previously validated with histology. ${ }^{15-17}$ In brief, SI relative to the adjacent sternocleidomastoid muscle from corresponding pixels in different contrast weightings of the same axial location was used to determine the presence/ absence of each feature as follows: 1) LRNC: isointense to hyperintense SI on T1-weighted, no contrast enhancement on CE-T1weighted, hypointense SI on T2-weighted images; 2) IPH: hyperintense SI on T1-weighted and TOF images; 3) calcification: hypointense SI on T1-weighted, T2-weighted, PDW, TOF, and CET1-weighted images; and 4) fibrous cap rupture: irregular lumen boundary, absence of a fibrous cap on CE-T1-weighted, and juxtaluminal IPH on TOF images. The proportion of each component (LRNC, calcification, and IPH), when present, relative to the wall volume was also calculated (eg, percenage LRNC volume $=$ LRNC volume/wall volume $\times 100 \%)$.

\section{Data Analysis}

Statistical analysis was performed only on arteries with $\leq 0 \%$ stenosis by MRA. Summary statistics for each metric are presented as mean \pm $\mathrm{SD}$ and the range. Histogram analysis is also provided to characterize the variability of disease within this particular cohort. The prevalence of plaque features (LRNC, IPH, calcification) and fibrous cap status was determined at the artery level and for 3 segments: 1) the common carotid artery: $>6 \mathrm{~mm}$ proximal to the flow divider; 2) the carotid bulb: $<0-6 \mathrm{~mm}$ proximal to the flow divider; and 3) the internal carotid artery: $\geq 0 \mathrm{~mm}$ distal to the flow divider.

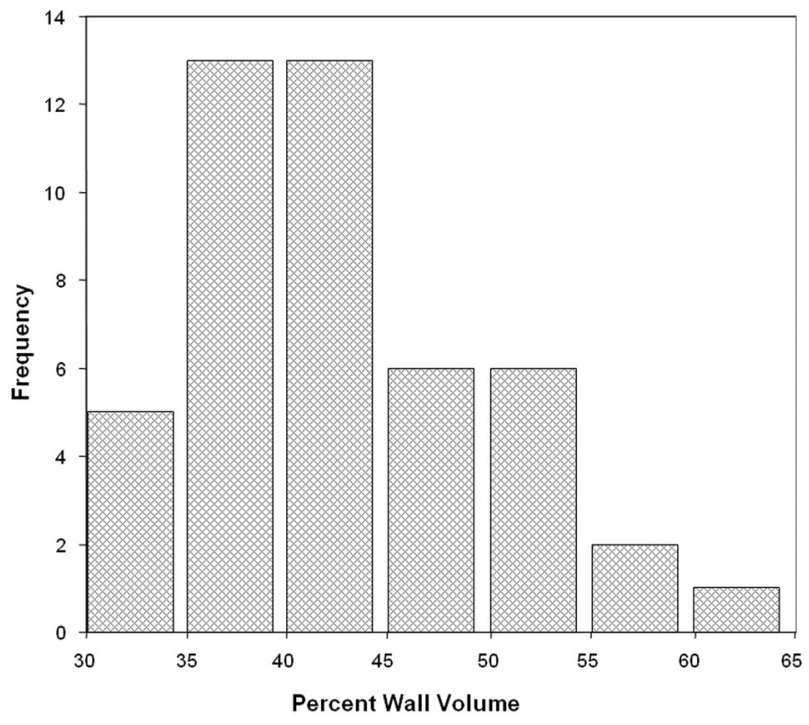

Fig 2. Histogram of plaque burden (percentage wall volume) for arteries with $0 \%$ stenosis on MRA.

\section{Results}

There were 144 arteries from 72 subjects available for review. Eleven $(7.6 \%)$ arteries had a noninterpretable MRA (motion artifacts, $n=4$; missed arterial phase with venous contamination, $n=4$; poor ImQ, $n=3$ ). Of the remaining 133 arteries, $36.1 \%$ (48/133) had 0\% stenosis on MRA. Of the 48 arteries with $0 \%$ stenosis by MRA, 95.8\% (46/48) had sufficient ImQ of $\geq 2$ on the corresponding multicontrast carotid MR imaging for interpretation of plaque morphology and composition. The demographic information for this subgroup $(n=46)$ is presented in the Table.

\section{Plaque Burden (Percentage Wall Volume)}

The mean \pm SD of percentage wall volume in arteries with $0 \%$ stenosis was $43.0 \pm 6.9 \%$, with a range from $31.6 \%$ to $60.1 \%$ (Fig 1). Histogram analysis (Fig 2) indicated that a substantial number of subjects with $0 \%$ stenosis had a percentage wall volume $>50 \%$.

\section{Plaque Composition}

A LRNC (Fig $1 B$ ) was present in $67.4 \%$ (31/46) of arteries, calcification was present in $65.2 \%(30 / 46)$, IPH was present in $8.7 \%(4 / 46)$, and fibrous cap rupture was present in $4.3 \%$ (2/46, Fig 3).

In arteries with an LRNC $(n=31)$, the average percentage LRNC volume was $8.8 \pm 7.3 \%$ with a range from $1.0 \%$ to $31.5 \%$. For calcification $(n=30)$, the mean percentage calcification volume was $3.8 \pm 4.2 \%$, with a range of $0.1 \%-17.4 \%$. The mean percentage IPH volume $(n=4)$ was $2.7 \pm 1.7 \%$, with a range of $0.5 \%-4.1 \%$. Histogram results for the volume of each plaque component are presented in Fig 4.

\section{Segmental Distribution of Plaque Features}

The prevalence of LRNC was highest in the carotid bulb (54.3\%, Fig 5), followed by the common carotid artery $(44.1 \%)$ and internal carotid artery $(37.0 \%)$. The prevalence of calcification was also highest in the carotid bulb (56.5\%), but prevalence in the internal carotid artery (37\%) was greater 

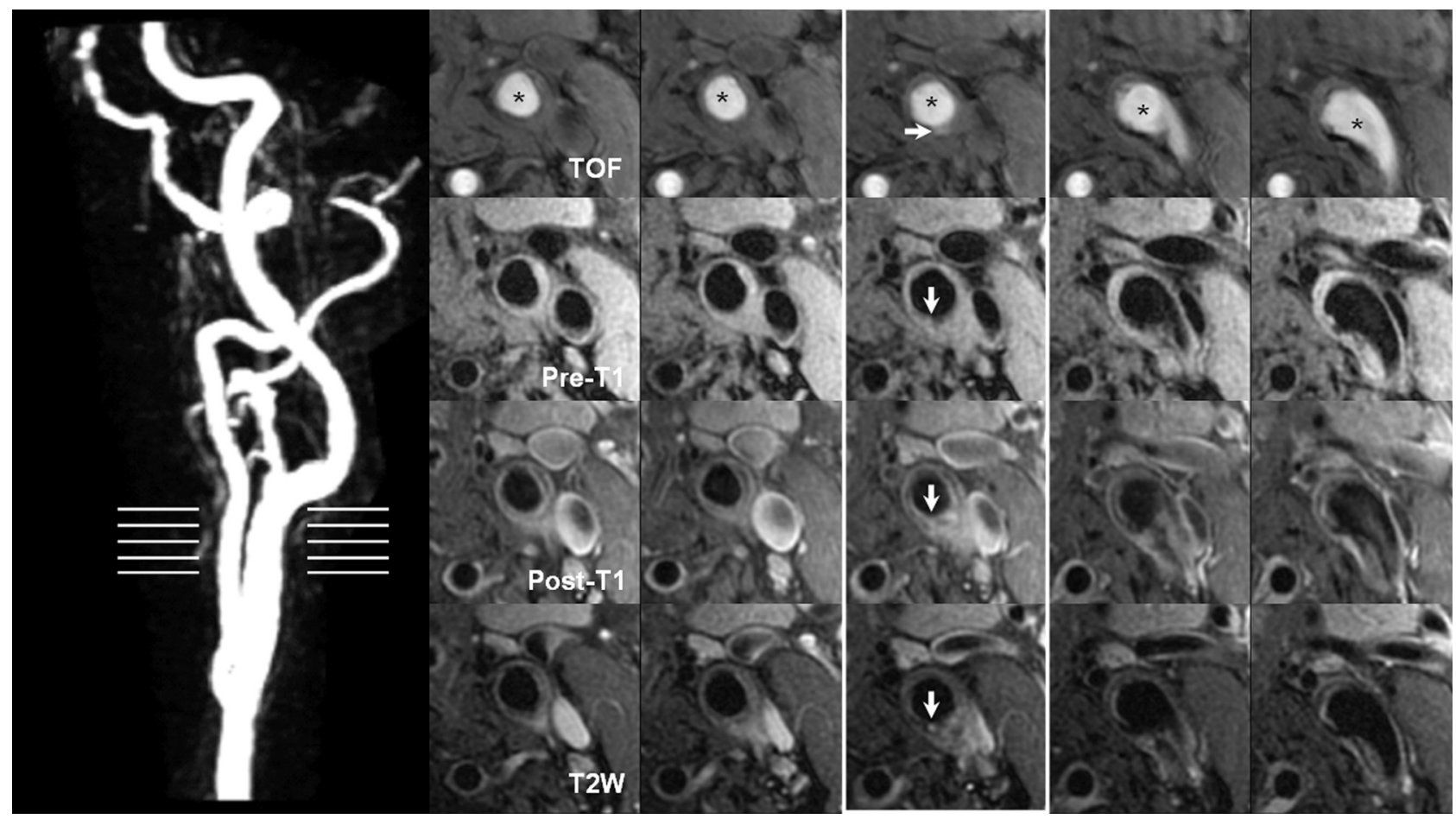

Fig 3. Transverse carotid plaque images and MIP of the left carotid artery were obtained from a 60-year-old woman. On the left side, MIP of the CE-MRA identifies a 0\% stenosis at the left CCA. The horizontal lines indicate the level of the transverse carotid plaque images as shown on the right side. On the transverse images, a surface disruption (white arrow) is noted as hyperintense on the TOF and hypointense on the pre- and postcontrast T1-weighted, T2-weighted, and proton-attenuation images. This surface disruption is not identified on CE-MRA. The asterisk indicates the lumen of either the CCA or ICA.

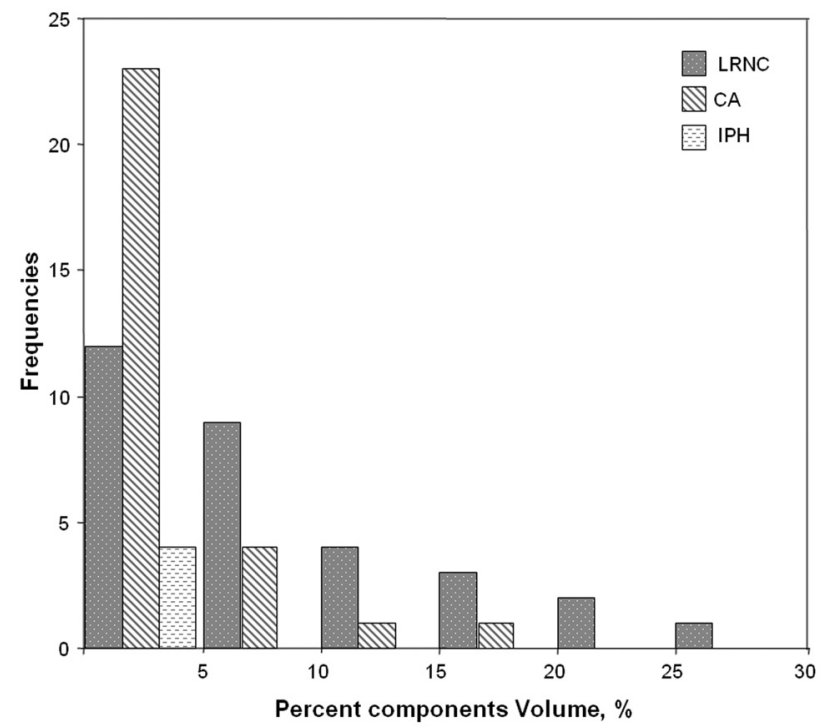

Fig 4. Histogram distribution for the size of each plaque component.

than that in the common carotid artery (26.5\%). IPH was not detected in the common carotid artery and had a similar prevalence in the internal carotid artery $(6.5 \%)$ and carotid bulb $(4.3 \%)$. In contrast, fibrous cap rupture was not present in the internal carotid artery and had a prevalence of $8 \%$ and $6.7 \%$ of lesions with an LRNC in the carotid bulb and common carotid artery, respectively.

\section{Discussion}

In this study of angiographically normal carotid arteries, a broad spectrum of atherosclerotic disease was identified.

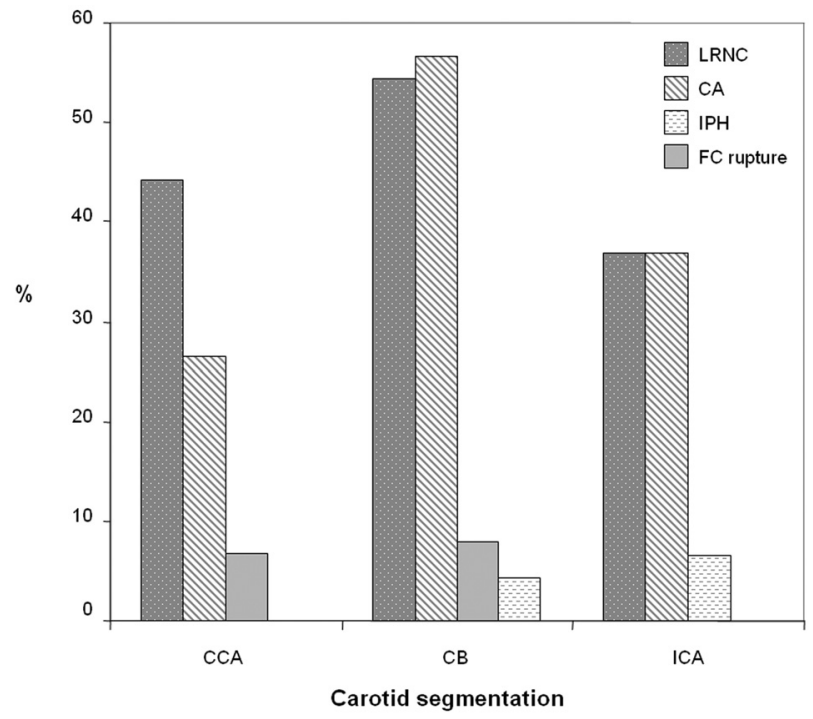

Fig 5. Prevalence of plaque features at each segment of the carotid artery. CCA: $>6 \mathrm{~mm}$ proximal to the flow divider; $\mathrm{CB}:>0-6 \mathrm{~mm}$ proximal to the flow divider; ICA: $\geq 0 \mathrm{~mm}$ distal to the flow divider.

Within this cohort, high-risk features such as IPH and fibrous cap rupture were not uncommon. However, given the extent of carotid disease in the contralateral artery $(>50 \%$ stenosis by duplex sonography), our findings should not be extrapolated to an at-large population of subjects with angiographically normal carotid arteries. Rather, these findings highlight the potential limitations of traditional risk-stratification systems that implement stenotic severity as the principal criterion. In addition, our findings offer compelling evidence for vessel 
wall imaging in patients with appropriate clinical risk factors and/or a history of neurologic events, but negative imaging findings based on conventional lumenography.

The occurrence of carotid atherosclerotic disease without luminal compromise may be attributable to several factors. In 1987, Glagov et $\mathrm{al}^{18}$ described a process of outward arterial remodeling in the coronary arteries. They observed that luminal narrowing did not occur until the atherosclerotic lesion occupied $>40 \%-50 \%$ of the internal elastic lamina area. ${ }^{18}$ In addition to this adaptive phenomenon, or separately, the morphology of the carotid bulb may contribute to the presence of lesions without angiographic changes. The transition between the common carotid artery and the flow divider (ie, the carotid bulb/sinus) is characterized by a localized dilation of the arterial lumen, which coincides with the presence of numerous baro- and chemoreceptors. Application of the NASCET criteria for measuring stenosis requires that a normal-appearing segment of the internal carotid artery be used as a reference. ${ }^{3}$ As such, in a normal artery, measurements from the carotid bulb compared with a reference segment of the internal carotid artery may yield a stenosis measurement $<0 \%$. We found that consistent with findings from a previous study, ${ }^{8}$ plaque compositional features occurred primarily at the carotid bulb. In addition, we found that a substantial number of subjects had a percentage wall volume $>50 \%$, which is the upper bound of the cutoff proposed by Glagov et $\mathrm{al}^{18}$ for the initiation of luminal narrowing. While these features may have resulted in luminal narrowing within the bulb consistent with the hypothesis of Glagov et al, the luminal diameter may have remained greater than the lumen of the internal carotid artery. An alternate approach to quantifying stenosis by angiography was used in the ECST. ${ }^{2}$ Stenosis was measured as the maximum percentage reduction in the relevant carotid artery. The relationship between the ECST and NASCET has been previously expressed by the following formula: ECST stenosis $=0.6 \times$ NASCET stenosis $+40 \% .{ }^{19}$ As such, this approach results in generally larger measures of stenosis than that by $\mathrm{NASCET}^{19}$ but requires extrapolation of where the nondiseased luminal boundary would be, which results in poor reader reproducibility. ${ }^{20}$ Collectively, these studies and the data presented herein offer compelling evidence that luminal stenosis alone may not be the strongest classifier of atherosclerotic disease severity.

A number of cross-sectional studies, both coronary and carotid, have indicated that fibrous cap rupture, IPH, and LRNC may represent the unstable atherosclerotic lesion. ${ }^{21-25}$ In the carotid artery, these features have been shown prospectively to be associated with a higher risk of plaque progression $^{26}$ and future cerebrovascular events. ${ }^{27}$ As such, vessel wall imaging may provide critical information for discerning lesion stability, particularly in all asymptomatic and symptomatic lesions with $<70 \%$ stenosis when treatment strategies remain ambiguous. Moreover, our data suggest that individuals with a history of an ischemic neurologic event may warrant carotid MR imaging in the absence of an identifiable etiology. In 1989, Sacco et $\mathrm{al}^{28}$ reported, in a prospective investigation of 1273 individuals with stroke, that $40 \%$ had infarcts of an undetermined cause. Subsequently, others have hypothesized that subclinical atherothromboembolism is the most likely etiology for cryptogenic stroke in older individuals or in patients with substantial atherosclerosis risk factors. ${ }^{29}$ The unique geometry of the carotid bulb and vascular remodeling may mask a stronger contribution to the etiology of stroke by the carotid artery than previously estimated by stenotic severity. Accordingly, vessel wall imaging may be necessary to better discriminate the origins of ischemic stroke.

\section{Conclusions}

Angiographically normal ( $0 \%$ stenosis) carotid arteries may have substantial plaque burden and a spectrum of compositional features, including IPH and fibrous cap rupture. Clinically, these findings indicate that vessel wall imaging may be necessary to better evaluate plaque stability across all levels of stenosis, particularly in patients with strong clinical risk factors. In addition, carotid MR imaging may be warranted in patients with angiographically normal carotid arteries and an unexplained etiology of ischemic stroke to rule out carotid atherosclerotic disease as a source of thromboemboli.

\section{References}

1. Moore WS, Barnett HJ, Beebe HG, et al. Guidelines for carotid endarterectomy: a multidisciplinary consensus statement from the Ad Hoc Committee, American Heart Association. Circulation 1995;91:566-79

2. Endarterectomy for moderate symptomatic carotid stenosis: interim results from the MRC European Carotid Surgery Trial. Lancet 1996;347:1591-93

3. Beneficial effect of carotid endarterectomy in symptomatic patients with high-grade carotid stenosis: North American Symptomatic Carotid Endarterectomy Trial Collaborators. N Engl J Med 1991;325:445-53

4. Clinical alert: benefit of carotid endarterectomy for patients with high-grade stenosis of the internal carotid artery-National Institute of Neurological Disorders and Stroke and Trauma Division. North American Symptomatic Carotid Endarterectomy Trial (NASCET) investigators. Stroke 1991;22: 816-17

5. Rothwell PM, Slattery J, Warlow CP. A systematic comparison of the risks of stroke and death due to carotid endarterectomy for symptomatic and asymptomatic stenosis. Stroke 1996;27:266-69

6. Rothwell PM, Gutnikov SA, Warlow CP. Reanalysis of the final results of the European Carotid Surgery Trial. Stroke 2003;34:514-23

7. Babiarz LS, Astor B, Mohamed MA, et al. Comparison of gadoliniumenhanced cardiovascular magnetic resonance angiography with highresolution black blood cardiovascular magnetic resonance for assessing carotid artery stenosis. J Cardiovasc Magn Reson 2007;9:63-70

8. Saam T, Underhill HR, Chu B, et al. Prevalence of American Heart Association type VI carotid atherosclerotic lesions identified by magnetic resonance imaging for different levels of stenosis as measured by duplex ultrasound. J Am Coll Cardiol 2008;51:1014-21

9. Yuan C, Kerwin WS, Yarnykh VL, et al. MRI of atherosclerosis in clinical trials. NMR Biomed 2006;19:636-54

10. Underhill HR, Yarnykh VL, Hatsukami TS, et al. Carotid plaque morphology and composition: initial comparison between 1.5- and 3.0-T magnetic field strengths. Radiology 2008;248:550-60

11. Yarnykh VL, Yuan C. T1-insensitive flow suppression using quadruple inversion-recovery. Magn Reson Med 2002;48:899-905

12. Yarnykh VL, Yuan C. Multislice double inversion-recovery black-blood imaging with simultaneous slice reinversion. J Magn Reson Imaging 2003;17: $478-83$

13. Kerwin W, Xu D, Liu F, et al. Magnetic resonance imaging of carotid atherosclerosis: plaque analysis. Top Magn Reson Imaging 2007;18:371-78

14. Nissen SE, Tuzcu EM, Schoenhagen P, et al. Effect of intensive compared with moderate lipid-lowering therapy on progression of coronary atherosclerosis: a randomized controlled trial. JAMA 2004;291:1071-80

15. Saam T, Ferguson MS, Yarnykh VL, et al. Quantitative evaluation of carotid plaque composition by in vivo MRI. Arterioscler Thromb Vasc Biol 2005; 25:234-39

16. Hatsukami TS, Ross R, Polissar NL, et al. Visualization of fibrous cap thickness and rupture in human atherosclerotic carotid plaque in vivo with highresolution magnetic resonance imaging. Circulation 2000;102:959-64

17. Cai J, Hatsukami TS, Ferguson MS, et al. In vivo quantitative measurement of intact fibrous cap and lipid-rich necrotic core size in atherosclerotic carotid plaque: comparison of high-resolution, contrast-enhanced magnetic resonance imaging and histology. Circulation 2005;112:3437-44

18. Glagov S, Weisenberg E, Zarins CK, et al. Compensatory enlargement of human atherosclerotic coronary arteries. N Engl J Med 1987;316:1371-75 
19. Rothwell PM, Gibson RJ, Slattery J, et al. Equivalence of measurements of carotid stenosis: a comparison of three methods on 1001 angiograms-European Carotid Surgery Trialists' Collaborative Group. Stroke 1994;25:2435-39

20. Griffiths GD, Razzaq R, Farrell A, et al. Variability in measurement of internal carotid artery stenosis by arch angiography and duplex ultrasonography: time for a reappraisal? Eur J Vasc Endovasc Surg 2001;21:130-36

21. Falk E. Why do plaques rupture? Circulation 1992;86:III30-42

22. Virmani R, Burke AP, Farb A, et al. Pathology of the vulnerable plaque. J Am Coll Cardiol 2006;47:C13-18

23. Kolodgie FD, Gold HK, Burke AP, et al. Intraplaque hemorrhage and progression of coronary atheroma. N Engl J Med 2003;349:2316-25

24. Yuan C, Zhang SX, Polissar NL, et al. Identification of fibrous cap rupture with magnetic resonance imaging is highly associated with recent transient ischemic attack or stroke. Circulation 2002;105:181-85
25. Murphy RE, Moody AR, Morgan PS, et al. Prevalence of complicated carotid atheroma as detected by magnetic resonance direct thrombus imaging in patients with suspected carotid artery stenosis and previous acute cerebral ischemia. Circulation 2003;107:3053-58

26. Takaya N, Yuan C, Chu B, et al. Presence of intraplaque hemorrhage stimulates progression of carotid atherosclerotic plaques: a high-resolution magnetic resonance imaging study. Circulation 2005;111:2768-75

27. Takaya N, Yuan C, Chu B, et al. Association between carotid plaque characteristics and subsequent ischemic cerebrovascular events: a prospective assessment with MRI-initial results. Stroke 2006;37:818-23

28. Sacco RL, Ellenberg JH, Mohr JP, et al. Infarcts of undetermined cause: the NINCDS Stroke Data Bank. Ann Neurol 1989;25:382-90

29. Kizer JR. Evaluation of the patient with unexplained stroke. Coron Artery Dis 2008;19:535-40 